\title{
Effectiveness of Pedestrian Safety Technologies for the New Age Car: A Review
}

\author{
Yusuf Idris Patrawala ${ }^{*}$, Rajashree D. Ghorpade ${ }^{\dagger}$ and Rahul G Gore ${ }^{\dagger}$ \\ ${ }^{\dagger}$ Mechanical Engineering Departement, Trinity College of Engineering \& Research (Savitribai Phule Pune University), Pune, India
}

Accepted 01 Sept 2016, Available online 02 Sept 2016, Vol.6, No.5 (Oct 2016)

\begin{abstract}
Road accidents are a human tragedy. Statistical figures demonstrate the need for an improved protection directed towards pedestrians, making the subject an upcoming field of research and development. This paper achieves its objective by comparing the ways in which automobiles can be designed to help reduce pedestrian fatalities, one being a world-first pedestrian airbag technology, addressing pedestrian protection in most situations when struck from the front end, consequently impacting onto the hood and the area around the windscreen-wiper recess and A-pillar, focus is made to help cushion the impact for a pedestrian by discussing the advanced technology along with various developments made in the design of the hood and introduction of an alternative to the front bumper system, economically allowing for sufficient safety. An overview of statistics, frequency of injury to different body parts, numerous tests and the complete chain of events from detection of a pedestrian to final state of deployment is studied. The methodology may be further applied in evaluating other vehicular technologies for traffic safety and the outcomes may effectively be utilized in establishing relevant traffic safety policies. In addition, some experimental concepts are presented along, to further increase the scope of the paper for future research.
\end{abstract}

Keywords: Pedestrian Safety, Passive Protection, Pedestrian Airbag, Future Airbag Technology, Advanced Vehicular Design.

\section{Introduction}

Over the years, year after year the vehicles have evolved from meagre wheels on a wooden board to pinnacles of power, efficiency, aesthetics, ergonomics and elegance. The safety of the vehicle and its occupants, since time long, has also been taken into serious consideration.

Researcher workers and engineers in the traffic safety field are actively interested in pedestrian to vehicle collision incidents now. Every year, many unprotected road users are fatally injured in road traffic accidents. In the European Union (EU), for example, 8000 unprotected bicyclists and pedestrians die, while 300,000 are injured severely and as much as 1.4 million are slightly injured (CARE, 2008), and 3000 pedestrian fatalities in Japan. In the United States, approximately 5000 pedestrian fatalities occur each year (2003-2012) (Yong Peng, 2012). In India, there were around 5 lakh road accidents, during the year 2010 , which resulted in the deaths of 134,513 people with pedestrians and others (bicyclists and twowheelers), comprising of the most unprotected road users, accounting for around $40 \%$ of all fatalities and out of the total 5 lakh road accident victims, 53.1\% were in the age group of 25 to 65 years (Pawan 2014).

*Corresponding author: Yusuf Idris Patrawala
Crash engineers have begun to use design principles that have been proved successful in protecting car occupants and significant efforts have been made worldwide to protect these vulnerable pedestrians against fatal collisions. Such technologies can primarily be classified into two vast research areas of, collision prevention and, mitigation of its severity. The former pertains to vehicular technology and involves systems of collision warning directed towards the driver. The latter, alternatively, is a technology applied during the occurrence of an accident (Cheol Oh, 2008).

This study is aimed to discuss a world first pedestrian airbag technology, being one way of directing towards pedestrian protection when struck from the front end, consequently impacting the hood and the recess area around the windscreen wiper and A-pillar. A description of the technology with evaluations of the technical performance, head impact characteristics and the overall performance of the technology is furnished from Lotta Jakobsson. Along with it, various other notable research to include suitable energy absorbers under the hood (Vesna Savic, 2014), the Active Hood Lift System (AHLS) (Cheol Oh, 2008) are studied, compared and the most suitable systems are combined to conceptually design the hood to absorb the upper body and head impacts to a certain extent before heading onto the external airbag. Also a 
combination of researches on designing of the bumper is studied replacing the standard energy absorbers with fibre reinforced epoxy composite and changing the shape to suite optimal energy absorbing capacities (M. M. Davoodi, 2007).

Various parameters like efficiency in protecting the pedestrian, complexity, replacement after use and cost, of all the systems, when used individually and when combined together, are studied to give an overview of the economy and feasibility of the system in various vehicles.

\section{Anatomy of a pedestrian-vehicle crash}

Most pedestrian vehicle crashes involve frontal impacts and the front structures of the vehicle are responsible for most pedestrian injuries (Fig. 1) (J R Crandall, 2002). In a frontal impact, the sequence of the crash scenario is well documented: the vehicle bumper contacts the lower limbs, the bonnet's leading edge strikes the proximal lower limb or pelvis, and, finally, the head and upper torso hit the top surface of the bonnet or windscreen(Fig. 2) (J R Crandall, et al, 2002).

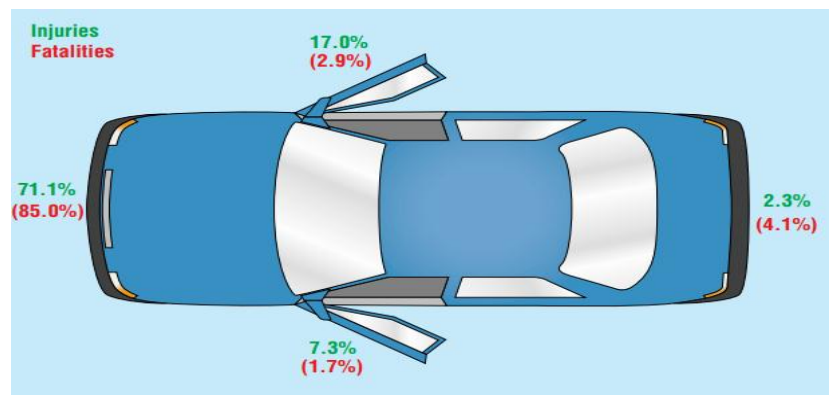

Fig. 1 Vehicle Impact Zones in Pedestrian-Vehicle Crashes (J R Crandall, 2002)

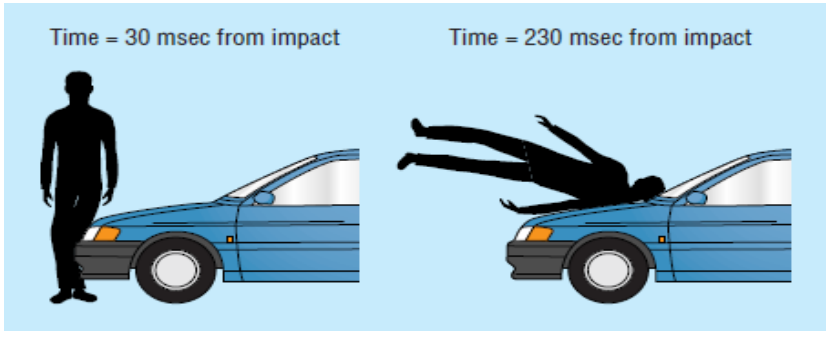

Fig. 2 Sequence of Events in a Pedestrian-Vehicle Crash (J R Crandall, 2002)

The pedestrians cross the street at low speeds and are subjected to impact speeds which increase the risk of severe injuries. The bumper forms the most frequent cause of injuries in accidents, causing $42.7 \%$ of all injuries. Other causes of pedestrian injuries in decreasing order are windscreen (30.7\%), bonnetedge (30\%) and bonnet (24.5\%) of all impacts (Yong Peng, 2012). Regarding the causes of head injuries, contact with the bonnet and windscreen (in order of occurrence) is the main factor (Yong Peng, 2012). And eventually, the pedestrians impact with the ground, often results in further injuries to the pedestrian.
Apparently, head collisions with windscreen frame and the locations close to the frame are more likely to result in serious injuries (AIS3+) (Yong Peng, 2012).

\subsection{Correlation between Head Injury Risk and Vehicle Impact Velocity}

For this part of the study, Y. Peng et al. 2012, used the method of logistic regression and generated S-shaped regression curves to illustrate the relationship (Fig. 3). In Fig. 3, the correlation between AIS2+ and AIS3+ head injury risks, and vehicle impact velocity, corresponding to Peng's calculation, is shown (Yong Peng, 2012). The 50\% probability of AIS2+ (moderate) head injury corresponds to vehicle impact velocity of $38.87 \mathrm{kmph}$ and that of AIS3+ (serious) head injury corresponds to vehicle impact velocity of $54.39 \mathrm{kmph}$ (Yong Peng, 2012). Based on the statistical results, in urban areas, the speed limit of the vehicles should be decidedin accordance with the accident frequency.

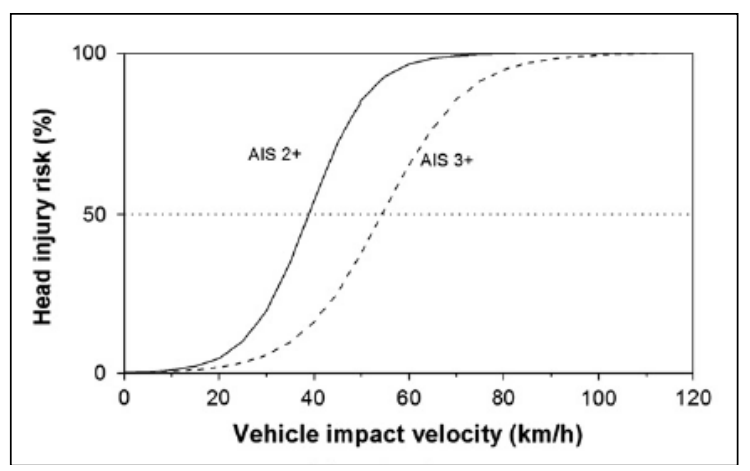

Fig. 3Logistic Regression Curve of Vehicle Impact Velocity for Head Injury Risk (Yong Peng, 2012)

In an area where frequency car-pedestrian accidents is considerably high, the speed limit should be $40 \mathrm{kmph}$. Higher vehicle impact speed produces higher head injury risk.

\section{Bumper design}

Bumper is the first component of the vehicle to come into contact and receives the collision when entering a crash situation (M M Davoodi, 2007), and which may to some extent protect the car body and passengers (M M Davoodi, 2012). The bumper system comprises three main parts: fascia, energy absorber, and the bumper beam integral with crush cans, given in order from the outside to inside (Fig. 4) (M M Davoodi, et al, 2012).

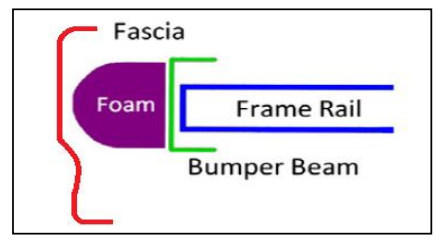

Fig. 4 Conventional Bumper System Schematic (M M Davoodi, 2012) 
The father is achieved by deflection of energy absorbers. It is reversible, in the sense that, it returns to approximately its original shape after deflection thus, dampening the collision (M M Davoodi, 2012). This is mainly described by the "bounce" of the material, and the restitution value of such components is equal to unity. Restitution is defined as the ability of a material to regain its original condition. Contrasting this, the latter is achieved by the deformation of the crush cans and the bumper beam to some extent, after their elastic limit is crossed. This deformation is irreversible and leads to permanent or plastic deformation of the component (M M Davoodi, 2012). The component is said to have "no bounce", and the restitution value is zero. The bumper also holds the sensors for the sensing of the collision.

(M M Davoodi, 2012) Two general approaches to reducing the pedestrian impacts were identified: (a) Using the bumper platform for impact sensors, which would differentiate a pedestrian impact from the others and deploy the exterior airbags and other safety measures studied further. (b) Provide cushioning and support of the lower limb with a energy absorber.

In this section, we will attempt to study the modification of the energy absorber by M M Davoodi et al. 2007, to extend the scope of the bumper system for protection of just the car and its occupants, to pedestrian safety as well.

The bumper system is designed for damping the kinetic energy without any damage to the vehicle or any plastic deformation of the bumper itself in a low speed impact, and for energy dissipation sometimes through plastic deformation, in high speed impact conditions (M M Davoodi, 2012).

\subsection{Pedestrian Impact Test for Bumper}

The pedestrian impact test for bumper system focuses mainly on leg-form impact, explained as follows. A legform impactor is propelled toward a stationary vehicle's longitudinal velocity of $40 \mathrm{kmphparallel}$ to the vehicle's longitudinal axis. The test can be performed at any location across the 30 degree bumper corners. The acceptance criteria are illustrated in Fig.5. The maximum tibia acceleration criterion is intended towards preventing tibia fractures. The knee bend angle and shear deformation criteria are intended to prevent knee joint injuries such as intra-articular bone fractures andligament ruptures (M M Davoodi, 2012).

\subsection{Development of Design}

A series of experimental work were conducted by M M Davoodi, et al, 2007, for the design of the composite energy absorber.

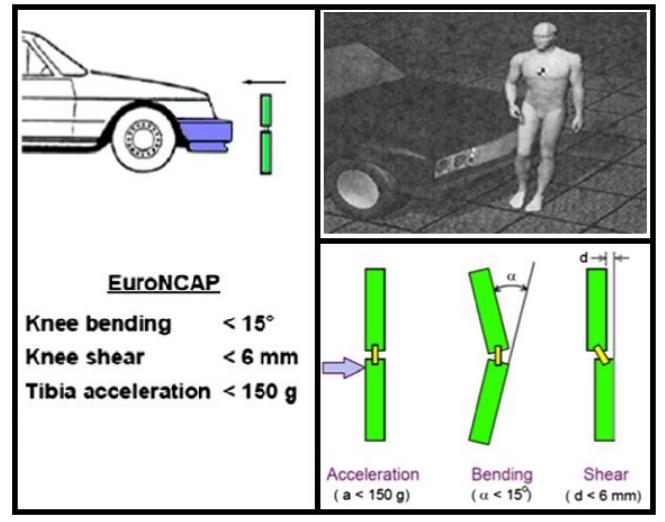

Fig. 5 Pedestrian 'Leg-form' Injury Criteria (M M Davoodi, 2012)

It can be seen from the graph in Fig.6 that the deflection increases to $46 \mathrm{~mm}$ and the load is $3000 \mathrm{~N}$. The graph shows two instances when the specimen was broken. Firstly, when the load was $600 \mathrm{~N}$ and the second, at approximately $3000 \mathrm{~N}$, it was broken completely (M M Davoodi, 2007).

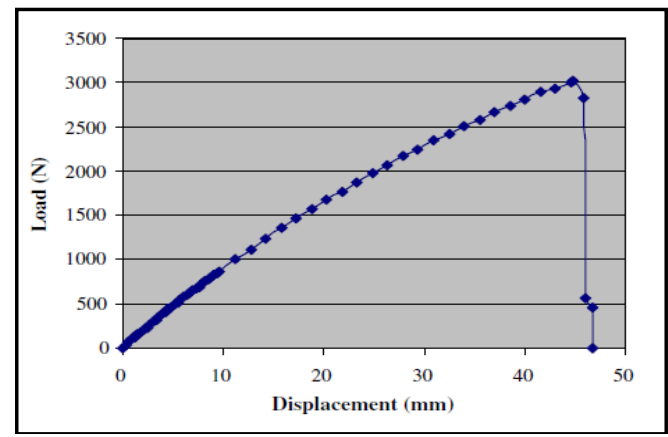

Fig. 6 Load vs. Displacement in Carbon Fibre Epoxy Composite (M M Davoodi, 2007)

In Fig.7, the accumulative energy absorption versus displacement of the specimen (carbon fibre reinforced epoxy composite) absorber by integrating load versus displacement equation from Fig.6. The energy absorption is 77J and the displacement is approximately $45 \mathrm{~mm}$ (M M Davoodi, 2007).

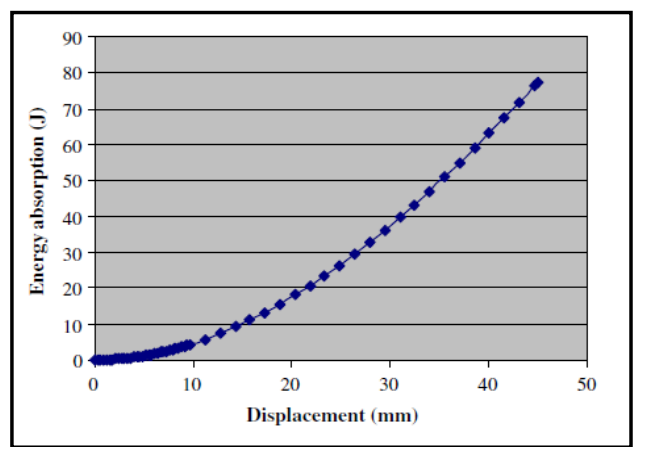

Fig. 7 Energy vs. Displacement of Carbon Fibre Epoxy Composite (M M Davoodi, 2007) 


\subsection{Calculations to Determine the Number of Composite Energy Absorbers}

The calculations are taken from the works conducted by Davoodi in their work et al. 2007.

According to the speed and the acceleration of the pedestrian leg-form discussed, we have $V=40 \mathrm{~km} / \mathrm{hr}$ (11.1 m/s) and a< 150g (see Fig.5) (M M Davoodi, 2007).

The total impact energy at $40 \mathrm{kmph}$ is,

$$
\begin{aligned}
& E_{c}=1 / 2 \mathrm{mv}^{2} \\
& E_{c}=1 / 2\left(13.4 \mathrm{~kg} \times 11.1^{2} \mathrm{~m} / \mathrm{s}\right) \\
& E_{c}=825 \mathrm{~J}
\end{aligned}
$$

The required force for leg-form can be obtained from Eq. (2) and the acceleration is less than 150g (see Fig.5)(M M Davoodi, 2007).Therefore,

$$
\begin{aligned}
& \mathrm{F}=\mathrm{m} \times \mathrm{a} \\
& \mathrm{F}=13.4 \mathrm{~kg} \times 150\left(9.8 \mathrm{~m} / \mathrm{s}^{2}\right) \\
& \mathrm{F}=19698 \mathrm{~N}
\end{aligned}
$$

The kinetic energy can be calculated from the integration of the load and displacement diagram,

$E_{c}=\int F \cdot d S$

Supposing less energy is lost on vibration in lowvelocity impact, then taking the impactor as a control volume, we obtain,

$$
825(\mathrm{~J})=19698(\mathrm{~N}) \times \mathrm{S}
$$

$\mathrm{S}=0.042 \mathrm{~m}$

If we assume the bumper skin shell and the steel mounting plate deflect in the direction of load, so we can consider that the load is distributed throughout the absorbers (M M Davoodi, 2007). Therefore, the required energy in the pedestrian leg - form (825 J) can be divided into energy absorption capacity (found from Fig.7) of the absorber and it is,

$825 / 77=10.7$

Therefore, 11 energy absorbers are required to satisfy the energy absorption of pedestrian leg-form (M M Davoodi, 2007).

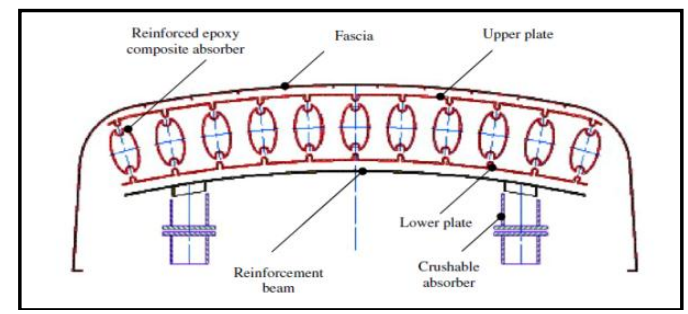

Fig. 8 Schematic View of Proposed Bumper Absorber (M M Davoodi, 2007)

\subsection{Final Design}

Based on the above analysis, it is evident that the standard EPP absorbers can be well substituted by fibre reinforced epoxy composite absorbers ( $M$ M Davoodi, 2007). Fig.8 shows the schematic drawing of the discussed design. In this figure, it can be seen that eleven fibre reinforced epoxy absorbers are encased between the bumper fascia and reinforcement beam(M M Davoodi, 2007). For mounting the absorbers, two knobby steel plates are placed on both sides of the absorber. The plates are fixed using by means of snap fits and rivets on the fascia and beam respectively (Fig.9) (M M Davoodi, 2007).

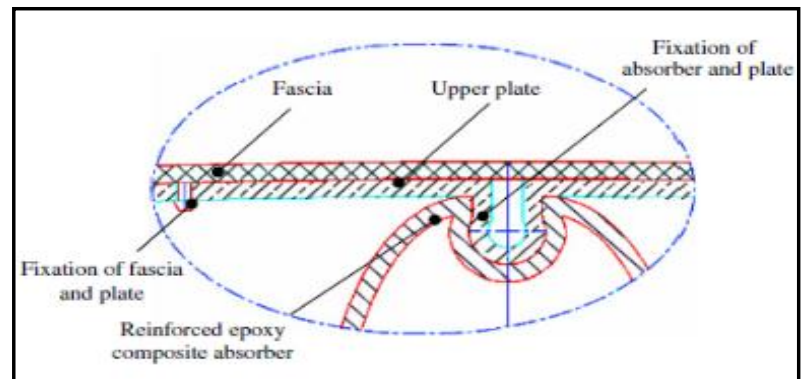

Fig. 9 Details of Fixation Method (M M Davoodi, 2007)

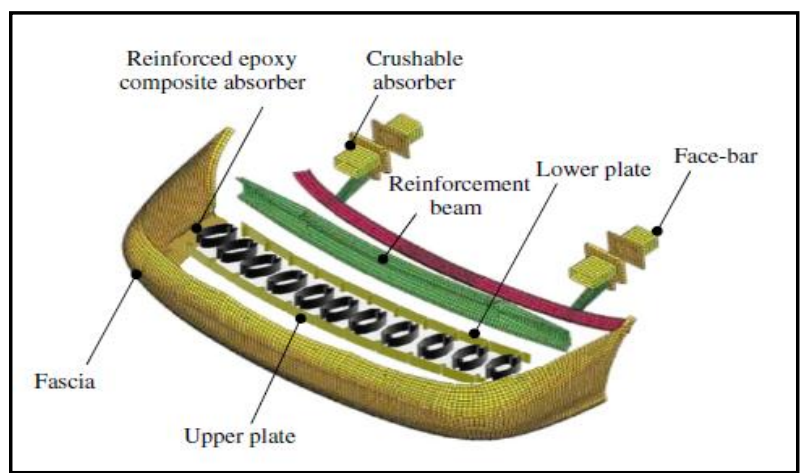

Fig.10 Final Design of the Proposed Bumper System(M M Davoodi, 2007)

\section{Hood Design}

The hood (or bonnet as it is known in many places) is the most vulnerable part of the vehicle front in terms of head injury in a pedestrian vehicle collision.Head injury is the main cause accounting for the fatality of the accident because of obvious reasons of the brain being sensitive, and that even a slight impact on the head area can lead to serious circumstances.

The hood must absorb a significant amount of energy over a small area while precluding impact with a hard engine compartment component, but there is limited space between the hood and the critical components under the hood, such as engine cover or washer fluid bottle due to demanding sleek design of the modern vehicles (Cheol Oh, et al, 2008).

\subsection{Head Injury Criterion (HIC)}

When the head of the pedestrian strikes the hood of a vehicle, the severity of the head injury is described by 
the Head Injury Criterion (Cheol Oh, 2008) and the value is called HIC score, which is a threshold for head injury in vehicle collisions (Vesna Savic, 2014). Injury is unlikely for HIC scores below an established threshold, while the likelihood of injury is greatest for HIC scores above an established threshold. The threshold value is mandated by different regulatory requirements, and that set by the EuroNCAP is 1000 .

The common method of evaluating the vehicle for pedestrian protection is to project a headform at the vehicle (Vesna Savic, 2014). The headform is usually instrumented with accelerometers that provide acceleration versus time data acquired during headform impact with a vehicle hood. With this the HIC score can be computed by,

$H I C=\left\{\left[\frac{1}{t_{2}-t_{1}} \int_{t_{1}}^{t_{2}} a(t) d t\right]^{2.5}\left(t_{2}-t_{1}\right)\right\}_{\max }$

where, $t_{2}-t_{1}$ is the HIC interval, the maximum value of which is $15 \mathrm{~ms}$ as mandated in the federal standard. Also, $\mathrm{a}(\mathrm{t})$ is the translational acceleration of the head center of gravity, $g=9.8 \mathrm{~m} / \mathrm{s}^{2}$, and the unit of the HIC score is second, although this is usually ignored (Cheol Oh, 2008).

\subsection{Active Hood Lift System (AHLS)}

The head of the pedestrian strikes the hood of the vehicle severely when a vehicle collides with the pedestrian at 30-40 km/hr collision speed (Cheol Oh, 2008). AHLS works by lifting up the vehicle's hood to obtain the space to absorb the impact energy just before the pedestrian's head hits the hood (Cheol Oh, 2008).

The system is composed of a module to detect a pedestrian or for that matter any impact over a threshold which may be harmful to the hit subject. Further, a hinge is provided on the front side of the hood, allowing to open up from the windscreen side and an actuator module that -deploys the lifting of the hood, as well as an electrical control unit (ECU) module to control the process from detection to deployment (See Fig.11) (Cheol Oh, 2008 and Lotta).
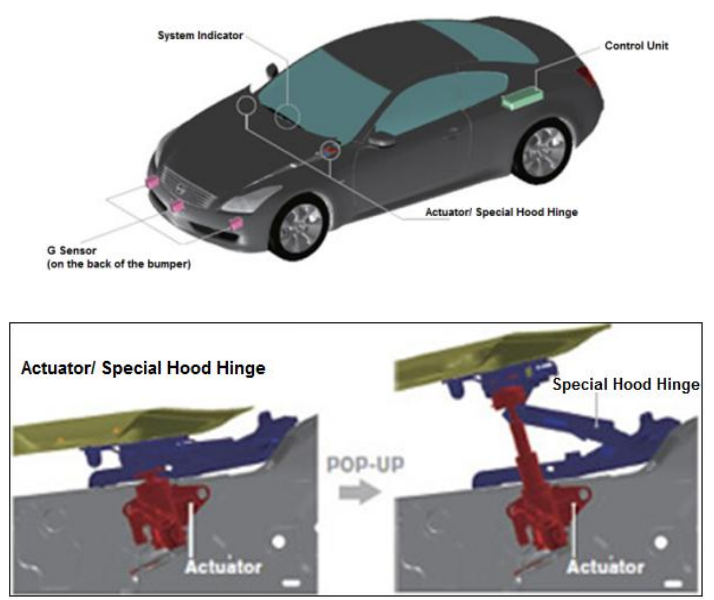

Fig. 11 The AHLS System and the Hood Hinge Mechanism
This system is a sub-system part of the Pedestrian Airbag Technology system studied in the later section, and thus a detailed study of the working and construction of this AHLS system is done there.

\subsection{Effectiveness of AHLS System in Reducing the Number of Fatalities}

Since AHLS reduces the value of HIC with respect to the head of a pedestrian in a collision with a vehicle, the change in number of fatalities due to the change in HIC can be regarded as the effectiveness of AHLS (Cheol Oh, 2008).

The relationship between pedestrian fatality and HIC cannot be directly obtained. The methodology proposed by Cheol Oh et. Al. 2008, consists of the following steps:

(a) A relation between collision speed and the pedestrian fatality is modelled.

(b) A relation between collision speed and HIC, is then established.

(c) Finally, the relation between pedestrian fatality and HIC is derived.

HIC measurements were carried out in the cases where AHLS was applied and not applied. The descriptive statistics on the value of HIC computed by the simulation are summarized in Table 1(Cheol Oh, 2008).

The HIC results from the experiments in LS-Dyna3D simulation show that the value of HIC decreased significantly when AHLS is applied (Cheol Oh, 2008)

Table 1HIC Values Computed from Simulation (Cheol, 2008)

\begin{tabular}{|c|c|c|c|c|c|}
\hline & N & HIC Values & & & \\
\hline & & Minimum & Maximum & Mean & $\begin{array}{c}\text { Standard } \\
\text { deviation }\end{array}$ \\
\hline $\begin{array}{c}\text { Without } \\
\text { AHLS }\end{array}$ & 24 & 910.0 & 4826.0 & 1928.6 & 990.5 \\
\hline With AHLS & 24 & 323.0 & 2190.0 & 931.4 & 392.9 \\
\hline
\end{tabular}

\subsection{Energy Absorbers under the Hood}

In this, a simple modification to the AHLS system is introduced by bending thin metal alloy sheets to follow a C-shaped cross-section profile, thereby giving them energy absorbing capacity during an impact when affixed to the underside of a hood (Fig.12). One restraint being the weight of the vehicle, the energy absorber material, shape and size selected should be optimized to make it feasible while not at all compromising the safety it is aimed to achieve (Vesna Savic, 2014). 


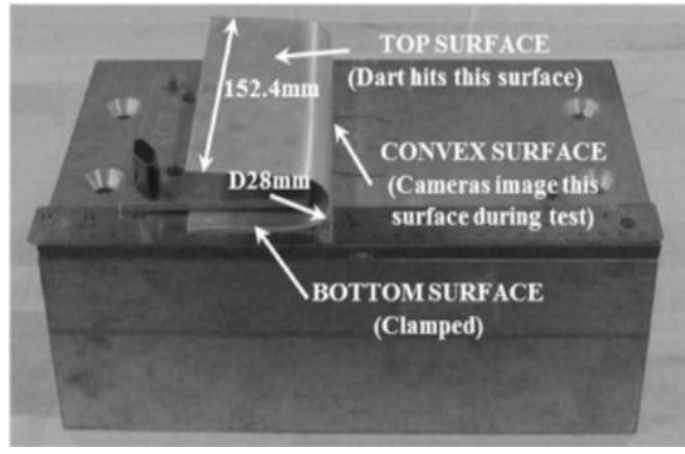

Fig. 12C-Channel Energy Absorber Clamped to a Steel Block with Main Dimensions (in mm) (Vesna Savic, 2014)

Materials selected for the purpose, studied from Matthew Pawlicki and Vesna Savic, et al. 2014 included a number of readily available lightweight alloys of aluminum (6111-T4, 5182-0) and magnesium (AZ31, AZ61, ZEK100) (Vesna Savic, 2014).

In their study, Matthew and Vesna developed a simple laboratory technique illustrated in Fig.13. Peak Velocity of $2.6-2.8 \mathrm{~m} / \mathrm{s}$ approximately was achieved, as measured just before the impact to energy absorber (Vesna Savic, 2014)

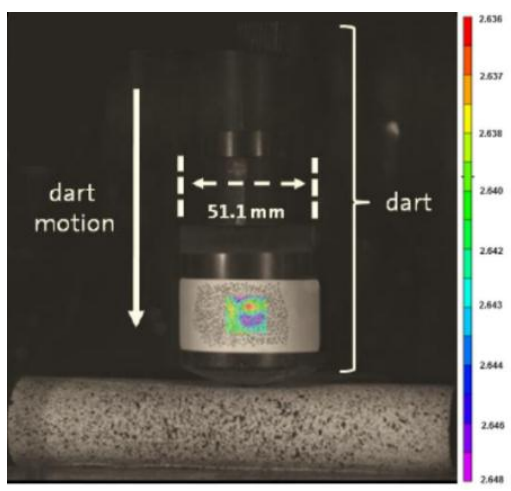

Fig. 13Impact of Dart at Contact with energy Absorber (Vesna Savic, 2014)

C-channel shaped specimens were chosen specially, based upon ease of fabrication, rapid installation in existing vehicle hoods, and ease of scalability to fit in tight spaces under the hood (Vesna Savic, et al, 2014).

\subsection{Results and Discussion of Dart Impact Tests}

\subsubsection{Load-Time Profiles during Impact Test}

Fig.14 and Fig.15 compares typical dart load (kN) vs. time (ms) records from impact tests of Al5182-0 (Fig.15) and Mg AZ31-0 (Fig.14). The profiles for other tested materials were similar to that of Al5182-0 (Vesna Savic, 2014).

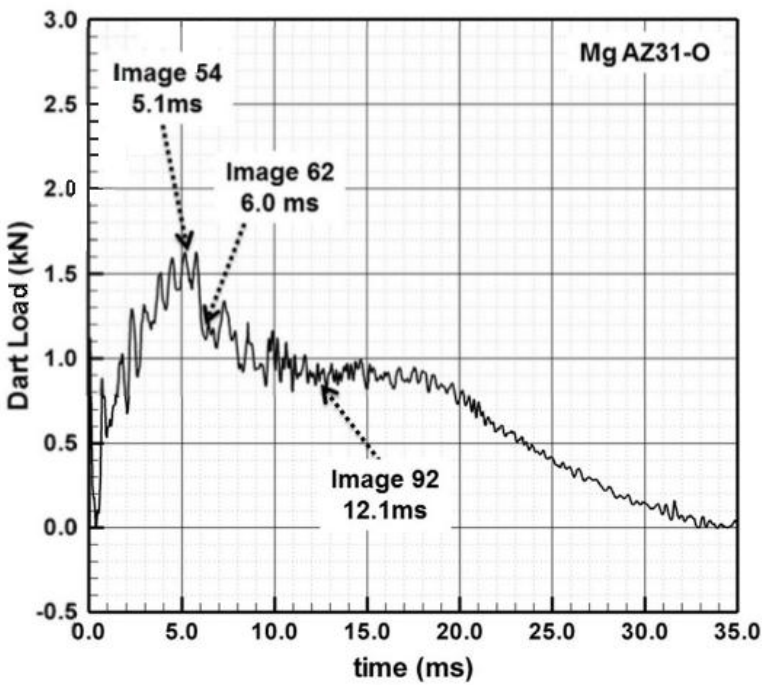

Fig. 14. Load Time Profile during Impact Test (Mg AZ31-0) (Vesna Savic, 2014)

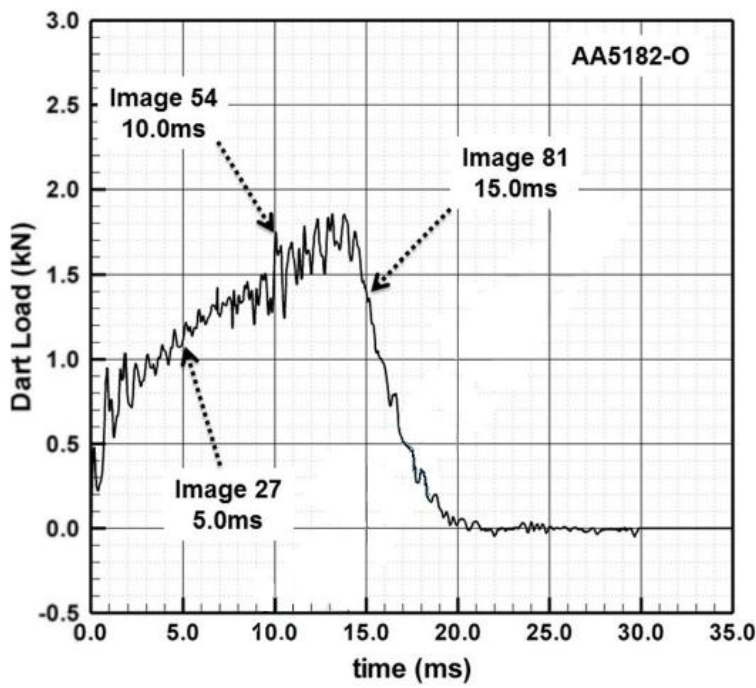

Fig. 15Load Time Profile during Impact Test (Al51820) (Vesna Savic, 2014)

Common to each profile in both the figures is a period early in the impact event during which the dart load rapidly rises. The peak dart load during impact is achieved at a later time in the test for Al5182-0 than for the Mg AZ31-0 material. Rather than quickly decreasing to zeroload once the peak load is achieved, as in the case for Al5182-0, the dart vs. time profile for the Mg AZ31-O material decreasing for a brief period only to level off in a "knee-shaped" region of the profile after approximately $10 \mathrm{~ms}$. This region extends to about $18 \mathrm{~ms}$ beyond which point, the curve gradually decreases to zero load as fracture ceases and the dart crushes the absorber (Vesna Savic, 2014).

\subsubsection{Velocity-Time Profiles during Impact Test}

Fig.16 and Fig.17 compares typical DIC-computed dart velocity $(\mathrm{m} / \mathrm{s})$ vs. Time $(\mathrm{ms})$ records from the impact tests of Al5182-O (Fig.17) and Mg AZ31-O (Fig.16) (Vesna Savic, 2014). 


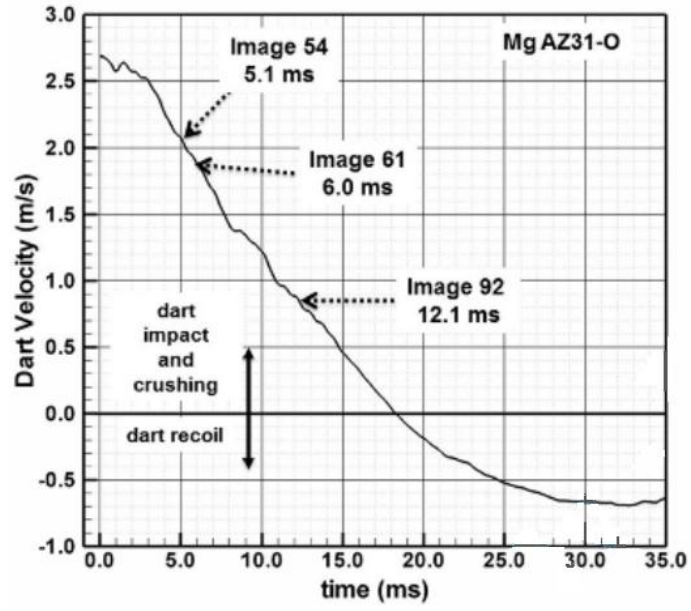

Fig. 16 Velocity Time Profile during Impact Test (Mg AZ31-0) (Vesna Savic, 2014)

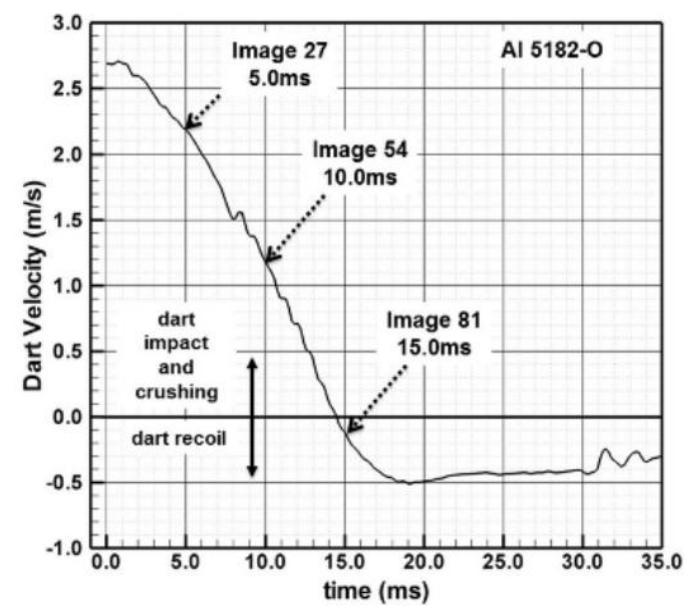

Fig. 17 Velocity Time Profile during Impact Test (Al5182-0) (Vesna Savic, 2014)

Positive velocity values denote dart impact with and subsequent crushing of the absorber, while negative values denote dart recoil. of particular interest is the time at which the dart velocity drops to zero in both figures. This signals the beginning of dart recoil. The dart velocity takes the longest time (about 18ms) to drop to zero in the figure for the Mg AZ31-O absorber. Alternatively, the dart achieves this at $14 \mathrm{~ms}$ for the Al5182-0 absorber (Vesna Savic, 2014).

\subsection{Images from Mg AZ31-O Dart Impact Test}

Figure 18shows images 92 and 54 respectively, denoted in Fig.14 and Fig.16 from the Mg AZ31-0 dart impact test. Image 54 corresponds to the peak dart load of about $16 \mathrm{kN}$ at $5.1 \mathrm{~ms}$. A crack first appeared close to the outer circumference of the dart during the impact at $6.0 \mathrm{~ms}$, approximately $1 \mathrm{~ms}$ after the peak load is reached. During the time when the load achieves a nearly constant value within the "kneeshaped" portion of the profile in Fig.14, energy absorption goes into crack growth along the convex surface of the energy absorber. The crack extended along the convex surface of the energy absorber in Fig.18at 12.1ms. Fig.19 shows the approximate $56 \mathrm{~mm}$ long crack in the energy absorber after the dart load is removed (Vesna Savic, 2014).

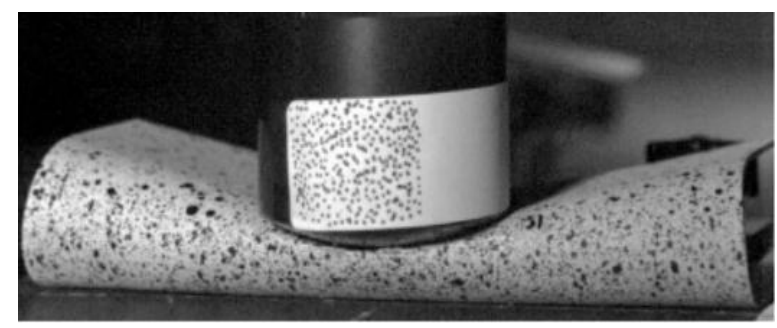

Fig. 18 Image 92 at 12.1ms (Vesna Savic, 2014)

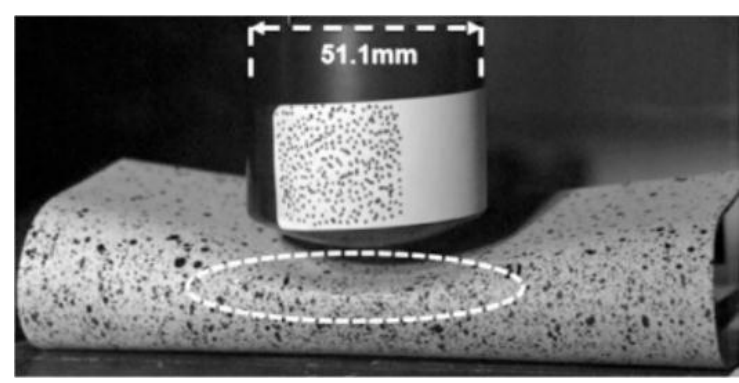

Fig.19 Digital Image showing an approximate $56 \mathrm{~mm}$ Crack (within white dashed ellipse) After the Dart Load is Completely Removed (Vesna Savic, 2014)

Fracture during the impact did not occur in any of the Mg AZ61-0, Al5182-0, ZEK100 or 6111-T4 energy absorber. It had the lowest of all, and the most favorable HIC score relative to those materials that crushed without fracturing (Vesna Savic, 2014).

\section{Pedestrian Airbag Technology}

Airbags have been since time long, used for the protection of the driver and other occupants of the vehicle, from all sides be it the front, the sides, the knees, and also behind the head. This successful piece of technology is now being redesigned and adapted to increase its function towards the protection of pedestrians in a collision.

The pedestrian airbag technology,being one possible solution to cushion an impact, help to protect pedestrians in certain situations when struck by the vehicle's front end with a consequent impact to the hood and the area around the windscreen, wiper recess and A-pillar (Lotta). It is a combination of the AHLS technology studied aboveand the existing airbag technology.

In this section, we will attempt to study a worldfirst pedestrian airbag technology offered in a production vehicle, being one way of addressing pedestrian protection (Fig.20) (Lotta). Sensors in the bumper provide input to the pedestrian airbag control unit that decides if the system should be activated. The pedestrian airbag deploys following the release of hood 
hinges,helping both to elevate the hood itself as well as helping to cushion a potential impact. The lift height is controlled and limited.

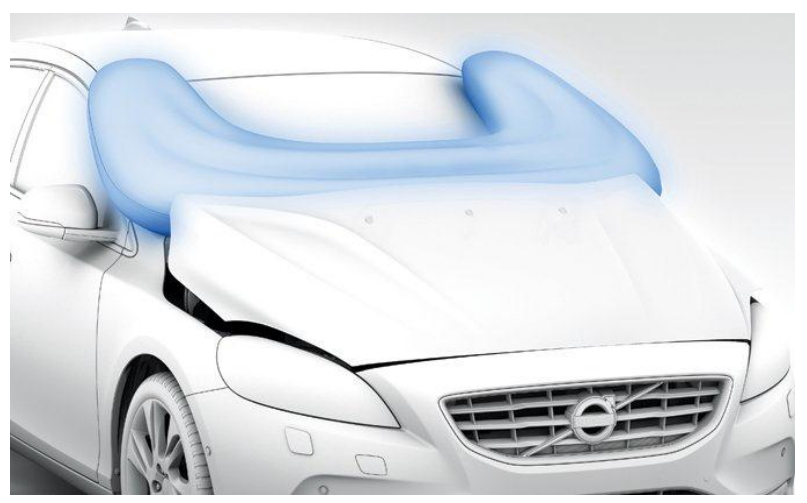

Fig. 20 The Pedestrian Airbag Technology Deployed

\subsection{Main Components and Working}

Fig.21 displays the main components of the pedestrian airbag technology, namely, sensors, hood hinge, hood hinge release mechanism, hood lift limiter, the pedestrian airbag control unit, pedestrian airbag module, in the order of activation (Lotta).

The system's activation range is between $20-50$ $\mathrm{km} / \mathrm{hr}$. Sensors embedded in the front of the car (bumper) transmit signals to the airbag control unit. When the car comes into contact with an object, the signal changes. The control unit assesses the signals and if it registers what it interprets as a human like leg, the pedestrian airbag technology is activated (Lotta).

When a decision to trigger the system is made, the trigger signals are simultaneously sent to the two hood hinge release mechanism and the air bagin flator. Each hood hinge release mechanism pulls out a pin which releases a second pivot point making it possible for the rear of the hood to lift. At the same time, the airbag starts filling with gas, opening the lid of the pedestrian airbag module cover. During this, the airbag raises the back edge of the hood to an extent of approximately ten centimetres. The lift height is controlled and limited by the hood lift limiter (Lotta).

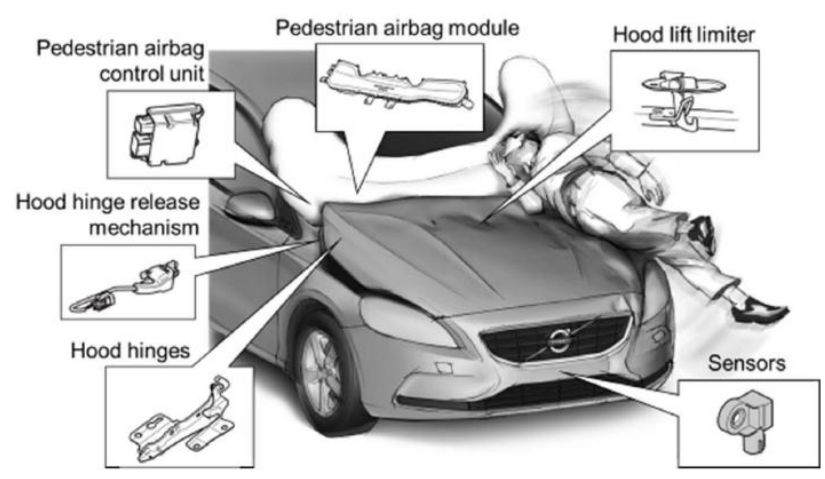

Fig. 21 Main Components of a Pedestrian Airbag Technology Integrated in the Vehicle (Lotta)
The hood lift limiter consists of a hook in the hood and a loop in the strut tower bar Fig.22. The hook is designed in a way, to hookinto the loop only when the back of the hood is lifted by the pedestrian airbag. Hence, when the hood is lifted in the front, for example, during servicing, the hook will not hitch into the loop (Lotta).

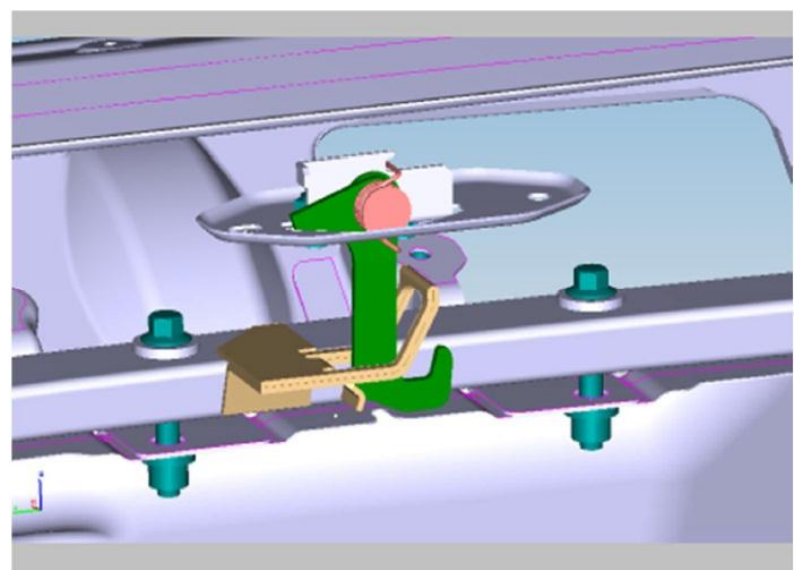

Fig. 22 Lift-Limiter Hook mounted on the Hood and Loop mounted on the Strut Tower Bar. (Lotta)

The sensors and the hood lifting mechanisms, i.e., the hood hinge release mechanism and the hood lift limiter is also the main parts of the AHLS system in the section above.

\subsection{Pedestrian Airbag Module}

Except for the airbag size and shape, the general concept is the same as the interior airbags, comprising of the cover, an inflator, and an airbag. Upon activation, the airbag is flushed with gas within few milliseconds (Lotta).

The entire sequence from activation of the system to full inflation and deployment of the airbag takes less than a hundred milliseconds. The total covering of the airbag corresponds to about one third of the windscreen (Lotta).

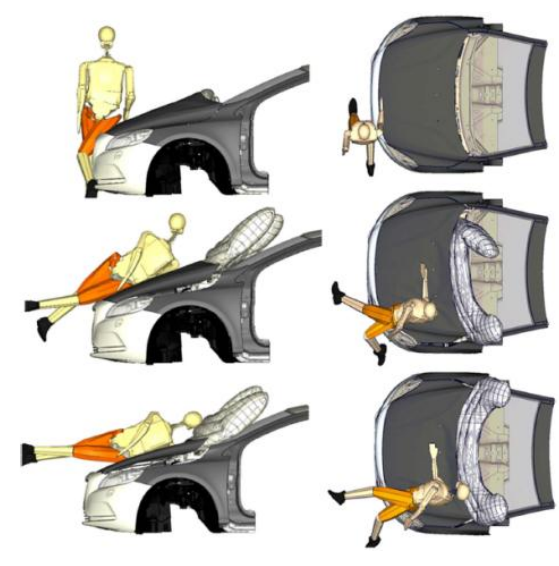

Fig. 23. Sequence from Activation to Full Inflation and Deployment of the Airbag in less than $100 \mathrm{~ms}$ (Lotta) 


\subsection{Head Impact Performance}

Head impact tests are performed according to the EuroNCAP Test Protocol. A child head impactor and an adult head impactor were used and launched towards the impact areas for the different pedestrian sizes. Used impact speed was $11.1 \mathrm{~m} / \mathrm{s}(40 \mathrm{~km} / \mathrm{hr})$. The injury criterion used for evaluation is the HIC (Head Injury Criterion), the value of which is based on the accelerometer signal from the head impactors (Lotta).

EuroNCAP grades the responses atvarious impact points in the scores of GOOD, ADEQUATE, and MARGINAL. The pedestrian airbag technology shows an overall GOOD performance and achieved a maximum score of 24 points on the head impact tests. The total pedestrian protection score was 88\% (Fig.24) (Lotta).

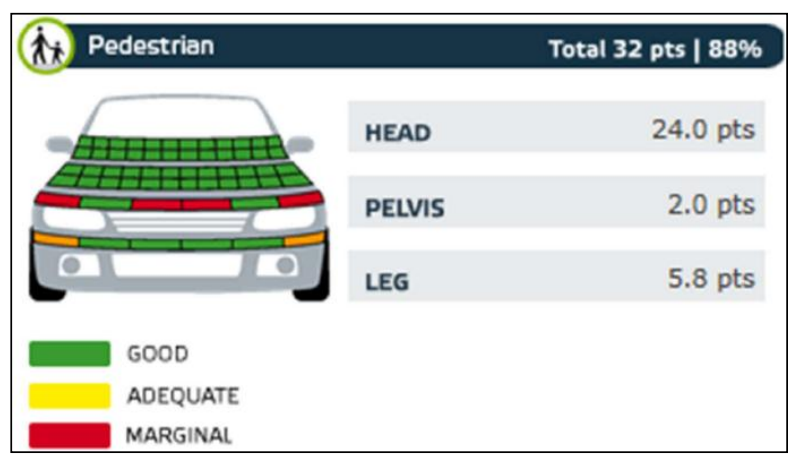

Fig. 24. EuroNCAP Results (Lotta)

An example of acceleration versus time signal for an impact point in the area of the pedestrian airbag is shown in Fig.25. A comparison is made at the same impact point, with and without an airbag. It can be seen that the pedestrian airbag extensively lowers the acceleration level and thus the HIC value (Lotta).

The increased distance against the underlying structure due to the lifted hood, provides further improvement regarding the Head Impact Performance, compared to the hood in its normal position (Lotta).

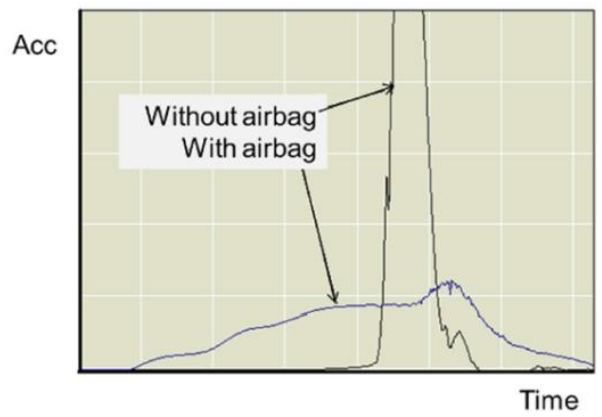

Fig.25.Head Impact Responses with and without Pedestrian Airbag (Lotta)

\subsection{Other Testing and Evaluation}

Numerous Computer Aided Engineering simulations were run by Lotta Jakkobson et al. 2012 to evaluate the performance of the entire system, including head impact timing, airbag coverage and overall kinematics (Lotta).

The human FE pedestrian models considered were of different sizes and also different pedestrian stances, impact points and collision speeds were considered(Lotta).

Physical testing was conducted to complement the CAE simulations. Overall kinematics criterion rather than a specific body region were considered and run at different speeds, dummy positions and vehicle impact points to add to the robustness of evaluation of the complete technology (Lotta).

Simulations were performed in various situations, including weather and loading conditions, and in different configurations to differentiate the activation due to human impact and several other environmental situations for the durability and functionality testing (Lotta).

The technology shows good performance in all of the evaluated configurations. Thus, the pedestrian airbag technology, comprising a pedestrian airbag along with a hood lifting functionality addresses improved protection to the pedestrian's upper body region (Lotta).

\section{Discussion}

In this study, a comparison was made, with reference to the statistical works produced by many known authors in this field, between the percentage fatalities of different road users when confronted with a vehicle in an event of an accident. A conclusion was thus drawn, that pedestrians were the most vulnerable in the event in general, as pedestrians cross the streets at lower speeds than any other road users like bicyclists, motorcycle riders, etc., which increases the risks of collisions. This was in turn due reason that, the drivers in the latter case, cannot get as much detection distance leading to insufficient deceleration times as compared to the case of other road users.

Fig.1shows that these vulnerable road users are mostly struck from the front end of a car as confirmed bythe finding of Crandall et al, 2002.The bumper is the most frequent cause of injuries, followed by the windscreen, bonnet edge, and bonnet (hood). The road is responsible for quite a rate too, for the injuries.

The bumper energy absorber has the main task in energy absorption in automotive bumper system. The impact severity with the bumper depends on its energy absorbing capacity, which in turn affects the injuries around the leg and knee region of the victim, the severity of the whiplash and, the projectile distance through which the pedestrian would be thrown across the road. Fibre Reinforced Polymer Composite material offers the needed characteristics such as weight reduction, flexibility in design and manufacturing and safety improvement. The energy absorbing capacity of the elliptical shaped composite absorber was found to be sufficient for a pedestrian impact along the bumper. 
The Active Hood Lift System can be introduced as an economical modification in a low range car to protect the pedestrian's torso and head to a great extent.Just before the pedestrian's head hits the hood,the AHLS works by lifting up the hood of a vehicle to obtain room to absorb the impact energy (Lotta). A simple modification to the AHLS system is studied by bending thin magnesium metal alloy (Mg AZ31-0) sheets to follow a C-shaped cross-section profile, thereby giving them energy absorbing capacity during an impact when affixed to the underside of a hood.

Pedestrian Airbag Technology is attempt that could be applied in very high end vehicles or in vehicles used in accident prone areas, to address pedestrian safety in a more advanced way. It is a combination of the AHLS technology and the existing airbag technology. Sensors in the bumper provide input to the pedestrian airbag control unit that determines if the system should be triggered. The hood hinges are released and the pedestrian airbag deploys, helping thus, to elevate the hood itself and moreover, helping to cushion a potential impact.

From the systems listed above, the Active Hood Lift System (AHLS), is the cheapest and can be applied to any low end vehicle easily. Followed by it is the AHLS System with Energy Absorber under the Hood, the Modified Bumper System and, the Pedestrian Airbag Technology in increasing order of their cost.The problems faced by the Pedestrian Airbag technology is that, once deployed, the airbag has to be replaced, increasing the cost of the vehicle and also the sensor systems and image processing should be advanced in the prediction of a pedestrian over other objects in an impact to get deployed.

\section{Conclusion}

Pedestrian-Vehicle collisions befalls pedestrians a high fatality rate. Thus, various countermeasures to prevent accidents involving pedestrians and to lessen the severity of such accidents needs to be devised. This study focused on the injury reducing capability of three systems, the modified bumper energy absorber, the Active Hood Lift System (AHLS), and the pedestrian airbag technology. Also an introduction of the energy absorbing hood was studied as an extension of the AHLS.
All these systems are aimed at only mitigation of accident severity to the pedestrian by reducing the impact velocity and acceleration, and absorbing to quite an extent, the impact energy during a collision. The new safety regulations and environmental legislations make the designing and integration of such vehicles quite complex, increasing the cost for the end user. From this review, it is concluded that by the introduction of any one of the less costly systems in the economic vehicles, we will be able to reduce the probability of fatality and save many costlier lives.

\section{References}

Peng Y., Chen Y., Jikuang Yang, Dietmar Otte, Remy Willinger (2012), A Study of Pedestrian and Bicyclist Exposure to Head Injury in Passenger Car Collisions Based on Accident Data and Stimulations, Safety Science 50, 2012, 17491759.

Deshpande Pawan (2014), Road Safety and Accident Prevention in India: A Review, Int J AdvEngg Tech/Vol $\mathrm{V} /$ Issue II.

Cheol Oh, Youn-soo Kang, Wonkyu Kim (2008), Assessing the Safety Benefits of an Advanced Vehicular Technology for Protecting Pedestrians, Accident Analysis and Prevention 40, 935-942.

Jakobsson Lotta, Broberg Thomos, Karlsson H., Anders Fredriksson, Niklas Graberg, Christina Gullander, Magdalena Lindman, Pedestrian Airbag Technology- A Production System, Volvo Car Corporation, Sweden, Paper Number 13-0447.

Savic Veena, Pawlicki M., Paul Krajewski, Mark Voss, Louis Hector, Keith Snavely (2014), Passive Pedestrian Protection Approach for Vehicle Hoods, 2014-01-0513.

Davoodi M. M., Sapuan S.M., Yunus R., Conceptual Design of a Polymer Composite Automotive Bumper Energy Absorber, Department of Mechanical and Manufacturing Engineering, Universiti Putra Malaysia, 43400 UPM Serdang, Selangor, Malaysia.

Crandall J. R., Bhalla K. S., Madeley N.J. (2002), Designing Road Vehicles for Pedestrian Protection, Centre for Applied Biomechanics, University of Virginia, 1011 Linden Avenue, Charlottesville, Virginia 22902, USA.

Davoodi M.M., Sapuan S.M., Aidy, Abu N.A. Osman, Oshkour A.A., Wan Abas W.A.B. (2012), Development Process of New Bumper Beam for Passenger Car: A Review, Materials and Design 40 304-313. 